\title{
Minimum Common String Partition Problem: Hardness and Approximations
}

\author{
Avraham Goldstein ${ }^{\star}$, Petr Kolman ${ }^{\star \star}$, and Jie Zheng ${ }^{\star \star \star}$
}

\begin{abstract}
String comparison is a fundamental problem in computer science, with applications in areas such as computational biology, text processing or compression. In this paper we address the minimum common string partition problem, a string comparison problem with tight connection to the problem of sorting by reversals with duplicates, a key problem in genome rearrangement.

A partition of a string $A$ is a sequence $\mathcal{P}=\left(P_{1}, P_{2}, \ldots, P_{m}\right)$ of strings, called the blocks, whose concatenation is equal to $A$. Given a partition $\mathcal{P}$ of a string $A$ and a partition $\mathcal{Q}$ of a string $B$, we say that the pair $\langle\mathcal{P}, \mathcal{Q}\rangle$ is a common partition of $A$ and $B$ if $\mathcal{Q}$ is a permutation of $\mathcal{P}$. The minimum common string partition problem (MCSP) is to find a common partition of two strings $A$ and $B$ with the minimum number of blocks. The restricted version of MCSP where each letter occurs at most $k$ times in each input string, is denoted by $k$-MCSP.

In this paper, we show that 2-MCSP (and therefore MCSP) is NP-hard and, moreover, even APX-hard. We describe a 1.1037-approximation for 2-MCSP and a linear time 4-approximation algorithm for 3-MCSP. We are not aware of any better approximations.
\end{abstract}

\section{Introduction}

String comparison is a fundamental problem in computer science, with applications in areas such as computational biology, text processing or compression. Typically, a set of string operations is given (e.g., delete, insert and change a character, move a substring or reverse a substring) and the task is to find the minimum number of operations needed to convert one string to the other. Edit distance or permutation sorting by reversals are two well known examples. In this paper we address, motivated mainly by genome rearrangement applications, the minimum common string partition problem (MCSP). Though MCSP takes a static approach to string comparison, it has tight connection to the problem of sorting by reversals with duplicates, a key problem in genome rearrangement.

* Email: avi_goldstein@netzero.net

** Institute for Theoretical Computer Science, Charles University, Malostranské nám. 25, 11800 Praha 1, Czech Republic. Email: kolman@kam.mff.cuni.cz. Research done while visiting University of California at Riverside. Partially supported by project LN00A056 of MŠMT ČR, and NSF grants CCR-0208856 and ACI-0085910.

*** Department of Computer Science, University of California, Riverside, CA 92521. Email: zjie@cs.ucr.edu. Supported by NSF grant DBI-0321756. 
A partition of a string $A$ is a sequence $\mathcal{P}=\left(P_{1}, P_{2}, \ldots, P_{m}\right)$ of strings whose concatenation is equal to $A$, that is $P_{1} P_{2} \ldots P_{m}=A$. The strings $P_{i}$ are called the blocks of $\mathcal{P}$. Given a partition $\mathcal{P}$ of a string $A$ and a partition $\mathcal{Q}$ of a string $B$, we say that the pair $\pi=\langle\mathcal{P}, \mathcal{Q}\rangle$ is a common partition of $A$ and $B$ if $\mathcal{Q}$ is a permutation of $\mathcal{P}$. The minimum common string partition problem is to find a common partition of $A, B$ with the minimum number of blocks. The restricted version of MCSP where each letter occurs at most $k$ times in each input string, is denoted by $k$-MCSP. We denote by $\#$ blocks $(\pi)$ the number of blocks in a common partition $\pi$. We say that two strings $A$ and $B$ are related if every letter appears the same number of times in $A$ and $B$.

In this paper, we show that 2-MCSP (and therefore MCSP) is NP-hard and, moreover, even APX-hard. We also describe a 1.1037-approximation for 2-MCSP and a linear time 4-approximation algorithm for 3-MCSP. We are not aware of any better approximations.

The signed minimum common string partition problem (SMCSP) is a variant of MCSP in which each letter of the two input strings is given a "+" or "-" sign. For a string $P$ with signs, let $-P$ denote the reverse of $P$, with each sign flipped. A common partition of two signed strings $A$ and $B$ is the pair $\pi=\langle\mathcal{P}, \mathcal{Q}\rangle$ of a partition $\mathcal{P}=\left(P_{1}, P_{2}, \ldots, P_{m}\right)$ of $A$ and a partition $\mathcal{Q}=\left(Q_{1}, Q_{2}, \ldots, Q_{m}\right)$ of $B$ together with a permutation $\sigma$ on $[m]$ such that for each $i \in[m]$, either $P_{i}=Q_{\sigma(i)}$, or $P_{i}=-Q_{\sigma(i)}$. All of our results apply also to signed MCSP.

Related work. 1-MCSP coincides with the breakpoint distance problem of two permutations [12] which is to count the number of pairs of symbols that are adjacent in the first string but not in the other; this problem is obviously solvable in polynomial time. Similarly as the breakpoint distance problem does, most of the rearrangement literature works with the assumption that a genome contains only one copy of each gene. Under this assumption, a lot of attention was given to the problem of sorting by reversals which is solvable in polynomial time for strings with signs [9] but is NP-hard for strings without signs [3]. The assumption about uniqueness of each gene is unwarranted for genomes with multi-gene families such as the human genome [10]. Chen et al. [4] studied a generalization of the problem, the problem of signed reversal distance with duplicates (SRDD); according to them, SRDD is NP-hard even if there are at most two copies of each gene. They also introduced the signed minimum common partition problem as a tool for dealing with SRDD. Chen et al. observe that for any two related signed strings $A$ and $B$, the size of a minimum common partition and the minimum number of reversal operations needed to transform $A$ to $B$, are within a multiplicative factor 2 of each other. (In the case of unsigned strings, no similar relation holds: the reversal distance of $A=1234 \ldots n$ and $B=n \ldots 4321$ is 1 while the size of minimum common partition is $n-1$.) They also give a 1.5-approximation algorithm for 2-MCSP. They reduce the problem to a vertex cover problem (on 6-claw-free graphs) and show that an $\alpha$-approximation for minimum vertex cover yields an $\alpha$-approximation for MCSP. Christie and Irving [5] consider the problem of (unsigned) reversal distance with duplicates (RDD) and prove that it is NP-hard even for strings over binary alphabet. 
Chrobak et al. [6] analyze a natural heuristic for MCSP, the greedy ${ }^{1}$ algorithm: iteratively, at each step extract a longest common substring from the input strings. They show that for 2-MCSP, the approximation ratio is exactly 3 , for 4-MCSP the approximation ratio is $\Omega(\log n)$; for the general MCSP, the approximation ratio is between $\Omega\left(n^{0.43}\right)$ and $O\left(n^{0.67}\right)$. The same bounds apply for SMCSP.

Closely related is the problem of edit distance with moves in which the allowed string operations are the following: insert a character, delete a character, move a substring. Cormode and Muthukrishnan [7] describe an $O\left(\log n \log ^{*} n\right)$ approximation algorithm for this problem. Shapira and Storer [11] observed that restriction to move-a-substring operations only (instead of allowing all three operations listed above) does not effect the edit-distance of two strings by more than a constant multiplicative factor. Since the size of a minimum common partition of two strings and their distance with respect to move-a-substring operations differ only by a constant multiplicative factor, the algorithm of Cormode and Muthukrishnan yields an $O\left(\log n \log ^{*} n\right)$-approximation for MCSP.

\subsection{Preliminaries}

Throughout the paper, we assume that the two strings $A, B$ given as input to MCSP are related. This is a necessary and sufficient condition for the existence of a common partition.

Given a string $A=a_{1} \ldots a_{n}$, for the sake of simplicity we will use the symbol $a_{i}$ to denote two different things. First, $a_{i}$ may denote the specific occurrence of the letter $a_{i}$ in the string $A$, namely the occurrence on position $i$. Alternatively, $a_{i}$ may denote just the letter itself, without any relation to the string $A$. Which alternative we mean will be clear from context.

Common partitions as mappings. Given two strings $A=a_{1} \ldots a_{n}$ and $B=$ $b_{1} \ldots b_{n}$ of length $n$, a common partition $\pi$ of $A$ and $B$ can be naturally interpreted as a bijective mapping from $A$ to $B$ (that is, if $P_{1}, \ldots, P_{m}$ is the partition of $A$ and $Q_{1}, \ldots, Q_{m}$ is the partition of $B$ in $\pi$, then for each $j \in[m]$, the letters from $P_{j}$ are mapped from left to right to the corresponding $Q_{j^{\prime}}$ ), and this in turn as a permutation on $[n]$. With this understanding in mind, we say that a pair of consecutive positions $i, i+1 \in[n]$ is a break of $\pi$ in $A$ if $\pi(i+1) \neq \pi(i)+1$. In other words, a break is a pair of letters that are consecutive in $A$ but are mapped by $\pi$ to letters that are not consecutive in $B$. The number of breaks in $\pi$ will be denoted by \#breaks $(\pi)$.

Clearly, not every permutation on $[n]$ corresponds to a common partition of $A$ and $B$. We say that a permutation $\rho$ on $[n]$ preserves letters of $A$ and $B$, if $a_{i}=b_{\rho(i)}$, for all $i \in[n]$. Then, every letter-preserving mapping $\rho$ can be interpreted as a common partition $\rho$, and \#blocks $(\rho)=\#$ breaks $(\rho)+1$. On the

\footnotetext{
${ }^{1}$ Shapira and Storer [11] also analyzed the greedy algorithm and claimed an $O(\log n)$ bound on its approximation ratio; unfortunately, the analysis was flawed, it applies only to a special subclass of MCSP problems.
} 
other hand, for a common partition $\pi=\langle\mathcal{P}, \mathcal{Q}\rangle$ interpreted as a permutation, $\#$ \#blocks $(\pi) \geq \#$ breaks $(\pi)+1$ (the inequality is due to possible unnecessary breaks in $\pi$ ). Thus, the MCSP problem is to find a permutation $\pi$ on $[n]$ that preserves letters of $A$ and $B$ and has the minimum number of breaks. An alternative formulation is that the goal is to find a letter-preserving permutation that maps the maximum number of pairs of consecutive letters in $A$ to pairs of consecutive letters in $B$.

Common partitions and independent sets. Let $\Sigma$ denote the set of all letters that occur in $A$. A $d u o$ is an ordered pair of letters $x y \in \Sigma^{2}$ that occur consecutively in $A$ or $B$ (that is, there exists an $i$ such that $x=a_{i}$ and $y=a_{i+1}$, or $x=b_{i}$ and $\left.y=b_{i+1}\right)$. A specific duo is an occurrence of a duo in $A$ or $B$. The difference is that a duo is just a pair of letters whereas a specific duo is a pair of letters together with its position. A match is a pair $\left(a_{i} a_{i+1}, b_{j} b_{j+1}\right)$ of specific duos, one from $A$ and the other one from $B$, such that $a_{i}=b_{j}$ and $a_{i+1}=b_{j+1}$. Two matches $\left(a_{i} a_{i+1}, b_{j} b_{j+1}\right)$ and $\left(a_{k} a_{k+1}, b_{l} b_{l+1}\right), i \leq k$, are in conflict if either $i=k$ and $j \neq l$, or $i+1=k$ and $j+1 \neq l$, or $i+1<k$ and $\{j, j+1\} \cap\{l, l+1\} \neq \emptyset$. Informally, two matches are in conflict if they cannot be realized at the same time.

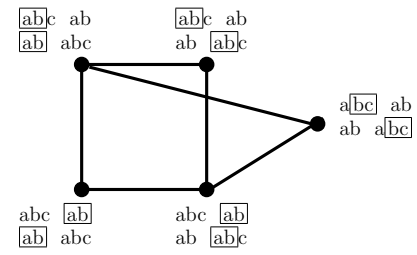

Fig. 1. Conflict graph for MCSP instance $A=a b c a b$ and $B=a b a b c$.

We construct a conflict graph $G=(V, E)$ of $A$ and $B$ as follows. The set of nodes $V$ consists of all matches of $A$ and $B$ and the set of edges $E$ consists of all pairs of matches that are in conflict. Figure 1 shows an example of a conflict graph. The number of vertices in $G$ can be much higher than the length of the strings $A$ and $B$ (and is trivially bounded by $n^{2}$ ).

Lemma 1. For $A=a_{1} \ldots a_{n}$ and $B=b_{1} \ldots b_{n}$, let $M I S(G)$ denote the size of the maximum independent set of the conflict graph $G$ of $A$ and $B$ and $m$ denote the number of blocks in a minimum common partition of $A$ and $B$. Then, $n-M I S(G)=m$.

Proof. Given an optimal solution for MCSP, let $S$ be the set of all matches that are used in this solution. Clearly, $S$ is an independent set in $G$ and $|S|=$ $n-1-(m-1)$.

Conversely, given a maximum independent set $S$, we cut the string $A$ between $a_{i}$ and $a_{i+1}$ for every specific duo $a_{i} a_{i+1}$ that does not appear in any match in 
$S$, and similarly for $B$. In this way, $n-1-|S|$ duos are cut in $A$ and also in $B$, resulting in $n-|S|$ blocks of $A$ and $n-|S|$ blocks of $B$. Clearly, the blocks from $A$ can be matched with the blocks from $B$, and therefore $m \leq n-|S|$.

Maximum independent set is an NP-hard problem, yet, two approximation algorithms for MCSP described in this paper make use of this reduction.

MCSP for multisets of strings. Let us consider a slight variant of the MCSP. Instead of two strings $A, B$, there are two multisets $\mathcal{A}, \mathcal{B}$ of strings on input. Similarly as before, a partition of the multiset $\mathcal{A}=\left\{A_{1}, \ldots, A_{l}\right\}$ is a sequence of strings $A_{1,1}, \ldots, A_{1, k_{1}}, A_{2,1}, \ldots, A_{2, k_{2}}, \ldots, A_{l, 1}, \ldots, A_{l, k_{l}}$, such that $A_{i}=A_{i, 1} \ldots, A_{i, k_{i}}$ for $i \in[l]$. For two multisets of strings, the common partition, the minimum common partition and the related-relation are defined similarly as for pairs of strings.

Let $\mathcal{A}=\left\{A_{1}, \ldots, A_{l}\right\}$ and $\mathcal{B}=\left\{B_{1}, \ldots, B_{h}\right\}$ with $h \leq l$, be two related multisets of strings, and let $x_{1}, y_{1}, \ldots, x_{l-1}, y_{l-1}$ be $2 l-2$ different letters that do not appear in $\mathcal{A}$ and $\mathcal{B}$. Considering two strings

$$
\begin{aligned}
& A=A_{1} x_{1} y_{1} A_{2} x_{2} y_{2} A_{3} \ldots x_{l-1} y_{l-1} A_{l}, \\
& B=B_{1} y_{1} x_{1} B_{2} y_{2} x_{2} B_{3} \ldots y_{h-1} x_{h-1} B_{h} y_{h} x_{h} \ldots y_{l-1} x_{l-1},
\end{aligned}
$$

it is easy to see that an optimal solution for the classical MCSP instance $A, B$ yields an optimal solution for the instance $\mathcal{A}, \mathcal{B}$ of the multiset version, and vice versa. In particular, if $m^{\prime}$ denotes the size of a MCSP of the two multisets of strings $\mathcal{A}$ and $\mathcal{B}$, and $m$ denotes the size of a MCSP of the two strings $A$ and $B$ defined as above, then

$$
m=m^{\prime}+2(l-1) .
$$

Thus, if one of the variants of the problems is NP-hard, so is the other.

\section{Hardness of approximation}

The main result of this section is the following theorem.

Theorem 1. 2-MCSP and 2-SMCSP are APX-hard problems.

We start by proving a weaker result.

Theorem 2. 2-MCSP and 2-SMCSP are NP-hard problems.

Since an instance of MCSP can be interpreted as an instance of SMCSP with all signs positive, and since a solution of SMCSP with all signs positive can be interpreted as a solution of the original MCSP and vice versa, it is sufficient to prove the theorems for MCSP only.

The proof is by reduction from the maximum independent set problem on cubic graphs (3-MIS) [8]. Given a cubic graph $G=(V, E)$ as an input for 3-MIS, for each vertex $v \in V$ we create a small instance $I_{v}$ of 2-MCSP. Then we process 
the edges of $G$ one after another, and, for each edge $(u, v) \in E$, we locally modify the two small instances $I_{u}, I_{v}$. The final instance of 2-MCSP, denoted by $I_{G}$, is the union of all the small (modified) instances $I_{v}$. We will show that a minimum common partition of $I_{G}$ yields easily a maximum independent set in $G$.

The small instance $I_{u}=\left(X_{u}, Y_{u}\right)$ for a vertex $u \in V$ is defined as follows (cf. Figure 2):

$$
\begin{aligned}
X_{u} & =\left\{d_{u}, a_{u} b_{u}, c_{u} d_{u} e_{u}, b_{u} e_{u} f_{u} g_{u}, f_{u} h_{u} k_{u}, g_{u} l_{u}, h_{u}\right\} \\
Y_{u} & =\left\{b_{u}, c_{u} d_{u}, a_{u} b_{u} e_{u}, d_{u} e_{u} f_{u} h_{u}, f_{u} g_{u} l_{u}, h_{u} k_{u}, g_{u}\right\}
\end{aligned}
$$

where $a_{u}, b_{u}, \ldots, l_{u}, a_{v}, b_{v}, \ldots, l_{v}$ are distinct letters in the alphabet. It is easy to check that $I_{u}$ has a unique minimum common partition, denoted by $O_{u}$, namely:

$$
\begin{aligned}
O_{u}= & \left\langle\left(d_{u}, a_{u} b_{u}, c_{u} d_{u}, e_{u}, b_{u}, e_{u} f_{u}, g_{u}, f_{u}, h_{u} k_{u}, g_{u} l_{u}, h_{u}\right)\right. \\
& \left.\left(b_{u}, c_{u} d_{u}, a_{u} b_{u}, e_{u}, d_{u}, e_{u} f_{u}, h_{u}, f_{u}, g_{u} l_{u}, h_{u} k_{u}, g_{u}\right)\right\rangle
\end{aligned}
$$

We observe that for $X_{G}=\bigcup_{u \in V} X_{u}$ and $Y_{G}=\bigcup_{u \in V} Y_{u}, I_{G}=\left(X_{G}, Y_{G}\right)$ is an instance of 2-MCSP, and the superposition of all $O_{u}$ 's is a minimum common partition of $I_{G}$. For the sake of simplicity, we will sometimes abuse the notation by writing $I_{G}=\bigcup_{u \in V} I_{u}$.

The main idea of the construction is to modify the instances $I_{u}$, such that for every edge $(u, v) \in E$, a minimum common partition of $I_{G}=\bigcup_{u \in V} I_{u}$ coincides with at most one of the minimum common partitions of $I_{u}$ and $I_{v}$. This property will make it possible to obtain a close correspondence between maximum independent sets in $G$ and minimum common partitions of $I_{G}$ : if $O_{v}$ denotes a minimum common partition of (the modified) $I_{v}$ and $O_{v}^{\prime}$ denotes the common partition of (the modified) $I_{v}$ derived from a given minimum common partition of $I_{G}$, then $U=\left\{u \in V \mid O_{u}^{\prime}=O_{u}\right\}$ will be a maximum independent set of $G$. To avoid the need to use different indices, we use $I_{G}$ to denote $\bigcup_{u \in V} I_{u}$ after any number of the local modifications; it will always be clear from context to which one are we referring.

For description of the modifications, a few terms will be needed. The letters $a_{u}$ and $c_{u}$ in $X_{u}$ are called left sockets of $I_{u}$ and the letters $k_{u}$ and $l_{u}$ in $X_{u}$ are right sockets. We observe that all the four letters $a_{u}, c_{u}, k_{u}, l_{u}$ appears only once in $X_{G}$ (and once in $Y_{G}$ ). Given two small instances $I_{u}$ and $I_{v}$ and a socket $s_{u}$ of $I_{u}$ and a socket $s_{v}$ of $I_{v}$, we say that the two sockets $s_{u}$ and $s_{v}$ are compatible,

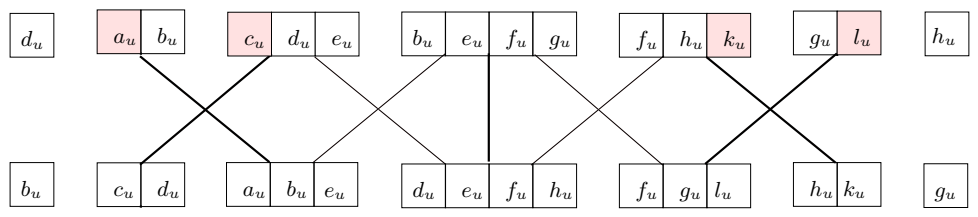

Fig. 2. An instance $I_{u}$ : the lines represent all matches, with the bold lines corresponding to the matches in the minimum common partition $O_{u}$. 
if one of them is a left socket and the other one is a right socket. Initially, all sockets are free.

For technical reasons, we orient the edges of $G$ in such a way that each vertex has at most two incoming edges and at most two outgoing edges. This can be done as follows: find a maximal set (with respect to inclusion) of edge-disjoint cycles in $G$, and in each cycle, orient the edges to form a directed cycle. The remaining edges form a forest. For each tree in the forest, choose one of its nodes of degree one to be the root, and orient all edges in the tree away from the root. This orientation will clearly satisfy the desired properties.

We are ready to describe the local modifications. Consider an edge $\overrightarrow{(u, v)} \in E$ and a free right socket $s_{u}$ of $I_{u}$ and a free left socket $s_{v}$ of $I_{v}$. That is, $R s_{u} \in X_{u}$ and $s_{v} S \in X_{v}$, for some strings $R$ and $S$. We modify the instances $I_{u}=\left(X_{u}, Y_{u}\right)$ and $I_{v}=\left(X_{v}, Y_{v}\right)$ as follows

$$
\begin{array}{ll}
X_{u} \leftarrow X_{u} \cup\left\{R s_{u} S\right\}-\left\{R s_{u}\right\}, & X_{v} \leftarrow X_{v} \cup\left\{s_{u}\right\}-\left\{s_{v} S\right\}, \\
Y_{u} \leftarrow Y_{u}, & Y_{v} \leftarrow Y_{v} \text { with } s_{v} \text { renamed by } s_{u} .
\end{array}
$$

(the symbols $\cup$ and - denote multiset operations).

After this operation, we say that the right socket $s_{u}$ of $I_{u}$ and the left socket $s_{v}$ of $I_{v}$ are used (not free). Note that in $Y_{v}$, the letter $s_{v}$ is renamed to $s_{u}$. All other sockets of $I_{u}$ and all other sockets of $I_{v}$ that were free before the operation remain free. We also note that $I_{u}$ and $I_{v}$ are not 2-MCSP instances. However, for every letter, the number of its occurrences is the same in $X_{G}$ and in $Y_{G}$, namely at most two. Thus, $I_{G}$ is still a 2-MCSP instance.

The complete reduction from a cubic graph $G=(V, E)$ to a 2-MCSP instance is done by performing the local modifications (4) for all edges in $G$.

\section{Reduction of 3-MIS to 2-MCSP}

1. $\forall u \in V$, define $I_{u}$ by the description (3),

2. $\forall \overrightarrow{(u, v)} \in E$, find a free right socket $s_{u}$ of $I_{u}$ and a free left socket $s_{v}$ of $I_{v}$, modify $I_{u}$ and $I_{v}$ by the description (4),

3. set $I_{G}=\bigcup_{u \in V} I_{u}$.

Since the in-degree and the out-degree of every node is bounded by two, and since every instance $I_{u}$ has initially two right and two left sockets, there will always be the required free sockets.

It remains to prove that a minimum common partition for the final $I_{G}$ (that is, when modifications for all edges are done) can be used to find a maximum independent set in $G$.

Lemma 2. Let $G$ be a cubic graph on $N$ vertices. Then, there exists an independent set $I$ of size $l$ in $G$ if and only if there exists a common partition of $I_{G}$ of size $12 N-l$.

Proof. Let $G_{C}$ be the conflict graph of $I_{G} ; G_{C}$ has $9 N$ vertices. The crucial observation is that each small instance $I_{u}$ can choose independently on all other small instances four of its nine possible matches in such a way that all these $4 N$ 
matches form an independent set in $G_{C}$ (in Figure 2, these four matches are represented by the thin lines). Let $O_{u}^{\prime}$ denote the four matches chosen by $u$.

Given an independent set $I$ of $G$, construct a common partition of $I_{G}$ as follows. For $u \in I$, use the five matches from $O_{u}$, and for $u \notin I$, use the four matches from $O_{u}^{\prime}$. The resulting solution will use $5 l+4(N-l)$ matches which corresponds to $9 N-(5 l+4(N-l))=5 N-l$ new breaks and $7 N+9 N-(5 l+$ $4(N-l))=12 N-l$ blocks.

Conversely, given a common partition of $I_{G}$ of size $m$, let $I$ consist of all vertices $u$ such that $I_{u}$ contributes 5 matches (i.e., 11 blocks) to the common partition. Then, $l \geq 12 N-m$, and the proof is completed.

Since the reduction can clearly be done in polynomial time (even in linear), with respect to $n=|V|$ and $m=|E|$, the proof of NP-hardness is completed.

Proof. (Theorem 1) Given a cubic graph $G$ on $N$ vertices, let $m^{\prime}$ denote the size of a minimum common partition of the instance $I_{G}=\left(X_{G}, Y_{G}\right)$ and let $m$ denote the size of a minimum common partition of the instance $(A, B)$, derived from the multiset instance $\left(X_{G}, Y_{G}\right)$ by definition 1 . We note that each of $X_{G}$ and $Y_{G}$ consists of $7 N-1$ strings. By Lemma 2 and relation (2), the size of a maximum independent set in $G$ is $5 N-m^{\prime}=19 N-2-m$, and an $\alpha$-approximation algorithm for MCSP instance $(A, B)$ can be used to derive an independent set in $G$ of size at least $19 N-2-\alpha \cdot m$.

Berman and Karpinski [2] proved that it is NP-hard to approximate 3-MIS within $\frac{140}{139}-\epsilon$, for every $\epsilon$. Thus, unless $\mathrm{P}=\mathrm{NP}$, for every $\epsilon>0$, the approximation ratio $\alpha$ of any algorithm for MCSP must satisfy

$$
\frac{19 N-2-m}{19 N-2-\alpha \cdot m} \geq \frac{140}{139}-\epsilon .
$$

Solving for $\alpha$ yields

$$
\alpha \geq \frac{19 N-2+139 m}{140 m}=1+\frac{19 N-2-m}{140 m} .
$$

Using the fact that the maximum independent set in $G$ has always size at lest $N / 4$ and therefore $m \leq 19 N-2-N / 4$, we conclude that it is NP-hard to approximate MCSP within $1+\frac{1}{75 \cdot 140}-\epsilon$, for every $\epsilon>0$.

Remark: To prove that only SMCSP is APX-hard, it is possible to start with smaller instances $I_{u}$ and thus get the constant larger.

\section{Algorithms}

\subsection{2-MCSP reduces to MIN 2-SAT}

Theorem 3. An $\alpha$-approximation algorithm for MIN 2-SAT yields $\alpha$-approximations for both 2-MCSP and 2-SMCSP. 
Plugging in a recent 1.1037-approximation algorithm of Avidor and Zwick [1], we get the following result.

Corollary 1. There exist polynomial 1.1037-approximation algorithms for 2MCSP and 2-SMCSP problems.

Proof. (Theorem 3) There are only minor differences between the reductions for signed and unsigned versions of the problem. We describe in detail the reduction for 2-MCSP and then briefly point out the differences for 2-SMCSP.

Let $A$ and $B$ be two related strings. We start the proof with two assumptions that will simplify the presentation:

(1) no duo appears at the same time twice in $A$ and twice in $B$, and that

(2) every letter appears exactly twice in both strings.

Concerning the first assumption, the point is that in 2-MCSP, the minimum common partition never has to break such a duo. Thus, if there exists in $A$ and $B$ such a duo, it is possible to replace it by a new letter, solve the modified instance and then replace the new letter back by the original duo. Concerning the other, a letter that appears only once can be replaced by two copies of itself. A minimum common partition never has to use a break between these two copies, so they can be easily replaced back to a single letter, when the solution for the modified instance is found.

The main idea of the reduction is to represent a common partition of $A$ and $B$ as a truth assignment of a (properly chosen) set of binary variables. With each letter $a \in \Sigma$ we associate a binary variable $X_{a}$. For each letter $a \in \Sigma$, there are exactly two ways to map the two occurrences of $a$ in $A$ onto the two occurrences of $a$ in $B$ : either the first $a$ from $A$ is mapped on the first $a$ in $B$ and the second $a$ from $A$ on the second $a$ in $B$, or the other way round. In the first case, we say that $a$ is mapped straight, and in the other case that $a$ is mapped across. Given a common partition $\pi$ of $A$ and $B$, if a letter $a \in \Sigma$ is mapped straight we set $X_{a}=1$, and if $a$ is mapped across we set $X_{a}=0$. In this way, every common partition can be turned into truth assignment of the variables $X_{a}, a \in \Sigma$, and vice versa. Thus, there is one-to-one correspondence between truth-assignments for the variables $X_{a}, a \in \Sigma$, and common partitions (viewed as mappings) of $A$ and $B$.

With this correspondence between truth assignments and common partitions, our next goal is to transform the two input strings $A$ and $B$ into a boolean formula $\varphi$ such that

- $\varphi$ is a conjunction of disjunctions (OR) and exclusive disjunctions (XOR),

- each clause contains at most two literals, and

- the minimal number of satisfied clauses in $\varphi$ is equal to the number of breaks in a minimum common partition of $A$ and $B$.

The formula $\varphi$ consists of $n-1$ clauses, with a clause $C_{i}$ for each specific duo $a_{i} a_{i+1}, i \in[n-1]$. For $i \in[n-1]$, let $s_{i}=1$ if $a_{i}$ is the first occurrence of the letter $a_{i}$ in $A$ (that is, the other copy of the same letter occurs on a position 
$i^{\prime}>i$ ), and let $s_{i}=2$ otherwise (that is, if $a_{i}$ is the second occurrence of the letter $a_{i}$ in $A$ ). Similarly, let $t_{i}=1$ if $b_{i}$ is the first occurrence of the letter $b_{i}$ in $B$ and let $t_{i}=2$ otherwise. We are ready to define $\varphi$. There will be three types of clauses in $\varphi$.

If the duo $a_{i} a_{i+1}$ does not appear in $B$ at all, we define $C_{i}=1$. The meaning is that in this case, $i, i+1$ is a break in $A$ in any common partition of $A$ and $B$. We call such a position an inherent break. Let $b$ be the number of clauses of this type.

If the duo $a_{i} a_{i+1}$ appears once in $B$, say as $b_{j} b_{j+1}$, let $Y=X_{i}$ if $s_{i} \neq t_{j}$, and let $Y=\neg X_{i}$ otherwise; similarly, let $Z=X_{i+1}$ if $s_{i+1} \neq t_{j+1}$ and let $Z=\neg X_{i+1}$ otherwise. We define $C_{i}=Y \vee Z$. In this way, the clause $C_{i}$ is satisfied if and only if $i, i+1$ is a break in a common partition consistent with the truth assignment of $X_{i}$ and $X_{i+1}$.

Similarly, if the duo $a_{i} a_{i+1}$ appears twice in $B$, we set $C_{i}=X_{i} \oplus X_{i+1}$ if $s_{i}=s_{i+1}$, and we set $C_{i}=\neg X_{i} \oplus X_{i+1}$ otherwise, where $\oplus$ denotes the exclusive disjunction. Again, the clause $C_{i}$ is satisfied if and only if $i, i+1$ is a break in a common partition consistent with the truth assignment of $X_{i}$ and $X_{i+1}$. Let $k$ denote the number of these clauses.

By the construction, a truth assignment that satisfies the minimum number of clauses in $\varphi=C_{1} \wedge \ldots \wedge C_{n-1}$ corresponds to a minimum common partition of $A$ and $B$. In particular, the number of satisfied clauses is equal to the number of breaks in the common partition which is by one smaller than the number of blocks in the partition.

The formula $\varphi$ resembles an instance of 2-SAT. However, 2-SAT formulas do not allow XOR clauses. One way to get around this is to replace every XOR clause by two OR clauses. This increases the length of the formula which in turn increases the resulting approximation ratio for 2-MCSP. In the rest of the section, we describe how to avoid this drawback.

Consider a duo $a_{i} a_{i+1}$ in $A$ for which $C_{i}$ is a XOR-clause. Then the duo $a_{i} a_{i+1}$ appears twice in $B$, and, by our assumption (1), the other occurrence of the letter $a_{i}$ in $A$ is followed by a letter different from $a_{i+1}$ (or the other occurrence of the letter $a_{i}$ is the last letter in $A$ ). This implies that $k \leq b+1$.

Let $\bar{\varphi}$ be the boolean formula derived from $\varphi$ by omitting clauses of the first type, that is, $\bar{\varphi}=\bigwedge_{i: C_{i} \neq 1} C_{i}$. Let $\varphi^{\prime}$ be the formula that we get from $\bar{\varphi}$ by replacing each XOR clause $(X \oplus Y)$ by $(X \vee Y) \wedge(\bar{X} \vee \bar{Y})$ and keeping all other clauses. Since for any values of boolean variables $X$ and $Y,(X \oplus Y)+1=$ $(X \vee Y)+(\bar{X} \vee \bar{Y})$, the minimal number of satisfied clauses in $\bar{\varphi}$ is exactly by $k$ smaller than the minimal number of satisfied clauses in $\varphi^{\prime}$.

Let $s$ be the minimal number of satisfied clauses in the formula $\bar{\varphi}$. Then, $s+b+1$ is the size of a minimum common partition of $A$ and $B$ and the minimal number of satisfied clauses in the 2-SAT formula $\varphi^{\prime}$ is $s+k$. An $\alpha$-approximation for MIN 2-SAT instance $\varphi^{\prime}$ satisfies at most $\alpha \cdot(s+k)$ clauses and the same truth assignment satisfies at most $\alpha \cdot(s+k)-k$ clauses in $\bar{\varphi}$. Considering the additional $b$ breaks for clauses of the first type, this truth assignment corresponds to a common partition with at most $\alpha \cdot(s+k)-k+b \leq \alpha \cdot(s+b+1)-1$ 
breaks. Since the size of the minimum common partition $s+b+1$, this is an $\alpha$-approximation. For unsigned MCSP, the proof is completed.

For signed MCSP, we use the same correspondence between truth assignments and common partitions; the only difference is in definition of the clauses $C_{i}$. We leave the details to the reader.

\subsection{Linear time 4-approximation for 3-MCSP}

In this section we exploit again the relation of MCSP and MIS in the conflict graph. The main idea of the algorithm is to cut the strings $A$ and $B$ into few pieces in such a way that the conflict graph of the modified instance becomes simple, making it possible to find MIS in polynomial time. More specifically, the desired property of $G$ is that the size of MIS equals the number of specific duos in (the modified) $A$. If this is the case, then MIS corresponds to a solution that does not need any (additional) breaks.

Similarly as in Section 3.1 we assume, without loss of generality, that no duo has three occurrences in $A$ and in $B$ at the same time. Again, the point is that if there exists a duo $a b$ with three occurrences in $A$ and three occurrences in $B$, for a 3 -MCSP instance $A, B$, the duo $a b$ never has to be broken in a minimum common partition of $A$ and $B$, and therefore can be replaced by a new letter $a^{\prime}$ without altering the size of the optimal solution. We augment both strings by a new character $a_{n+1}=b_{n+1}=\$$.

A duo $a b$ is good if the number of its occurrences in $A$ equals the number of its occurrences in $B$, and is bad otherwise. As before, let $m$ denote the size of a minimum common partition of a given pair of strings $A$ and $B$.

Observation 4 In every common partition of $A$ and $B$, for every bad duo ab there must be at least one break immediately after some occurrence of a in $A$ and at least one break immediately after some occurrence of a in B.

In the first phase of the algorithm, for every bad duo $a b$, we cut both strings $A$ and $B$ after every occurrence of $a$. We charge the cuts of $a b$ to the breaks that appear by Observation 4 in the optimal partition, that is, to the breaks after letter $a$. At most three cuts are charged to a single break. Let $\mathcal{A}$ and $\mathcal{B}$ denote the two multisets of strings we obtain from $A$ and $B$ after performing all these cuts.

At this point, every specific duo of $\mathcal{A}$ and $\mathcal{B}$ has either one or two matches. In the first case, we talk about a unique duo and a unique match, in the later case about an ambiguous duo and an ambiguous match. There are four vertices in the conflict graph corresponding to matches of an ambiguous duo and they are connected in a 4-cycle. The 4-cycle will be called a square $a b$; a vertex corresponding to a match of a unique duo will be called a single. We say that two duos interfere if a match of the first duo is in a conflict with a match of the other duo.

Let $G_{1}$ be the conflict graph of $\mathcal{A}$ and $\mathcal{B}$. The edges of $G_{1}$ can be classified into three groups: edges between two ambiguous matches, between an ambiguous 
match and a unique match, and, between two unique matches. We are going to have a closer look on these three cases.

Consider two ambiguous duos that interfere, say $a b$ and $b c$. There are only two ways how the two occurrences of $a b$ and the two occurrences of $b c$ can appear in $\mathcal{A}$ : either there are two occurrences of a substring $a b c$, or a single occurrence of each of $b c, a b c$ and $a b$, in any order. There are the same possibilities for $\mathcal{B}$. Thus, there are only three basic ways how the duos $a b$ and $b c$ can appear in the strings $\mathcal{A}$ and $\mathcal{B}$ (up to symmetry of $\mathcal{A}$ and $\mathcal{B}$ and up to permutation of the depicted substrings):

$$
\begin{aligned}
& \mathcal{A}=\ldots a b c \ldots a b c \ldots, \quad \mathcal{B}=\ldots a b c \ldots a b c \ldots \\
& \mathcal{A}=\ldots b c \ldots a b c \ldots a b \ldots, \quad \mathcal{B}=\ldots b c \ldots a b c \ldots a b \ldots \\
& \mathcal{A}=\ldots a b c \ldots a b c \ldots, \quad \mathcal{B}=\ldots b c \ldots a b c \ldots a b \ldots
\end{aligned}
$$

The corresponding subgraphs of $G_{1}$ are depicted in Figure 3 . We observe several things. If we want to keep all occurrences of $a b$ and $b c$ in case (1.1) and it is already given how to match the $a b$ duos ( $b c$, resp.), then it is uniquely given how to match the $b c$ duos ( $a b$, resp.). If we want to keep all occurrences of $a b$ and $b c$ in case (1.2), then there is only one way how to match them all; we say that the duos have a preference and we call these matches the preferred matches of $a b$ and $b c$. Concerning case (1.3), in any common partition of $\mathcal{A}$ and $\mathcal{B}$ at least one occurrence of the duos $a b$ and $b c$ must be broken.

Let $a b$ be an ambiguous duo and $b c$ a unique duo. There are two possibilities how they may interfere (cf. Figure 3):

$$
\begin{aligned}
\mathcal{A} & =\ldots a b c \ldots a b \ldots, \mathcal{B}=\ldots a b c \ldots a b \ldots \\
\mathcal{A} & =\ldots a b \ldots a b c \ldots, \mathcal{B}=\ldots a b \ldots a b \ldots b c \ldots
\end{aligned}
$$

Similarly as before, there is only one way how to match all duos in case (2.1). We sat that the duos have a preference and we call these matches the preferred matches of $a b$ and $b c$. Concerning (2.2), in any common partition of $\mathcal{A}$ and $\mathcal{B}$ at least one of $a b$ and $b c$ must be broken.

Finally, let $a b$ and $b c$ be two unique duos. There is only one way how they may interfere.

$$
\mathcal{A}=\ldots a b c \ldots, \mathcal{B}=\ldots a b \ldots b c \ldots
$$

Again, in any common partition of $\mathcal{A}$ and $\mathcal{B}, a b$ or $b c$ must be a break.

In the second phase of the algorithm, we cut all occurrences of duos $a b$ and $b c$ that have an interference of type (1.3), (2.2) or (3.1). We charge these cuts to the breaks that appear, by the above observations, in the optimal solution. At most four cuts are charged to a single break.

Let $\mathcal{A}_{2}$ and $\mathcal{B}_{2}$ denote the two sets of strings after performing all cuts of phase two, and let $G_{2}$ be the corresponding conflict graph. Note that a duo might have more than one preferred match. The problem we are facing now is to decide which preferences to obey and which not since the more preferences 
1.1
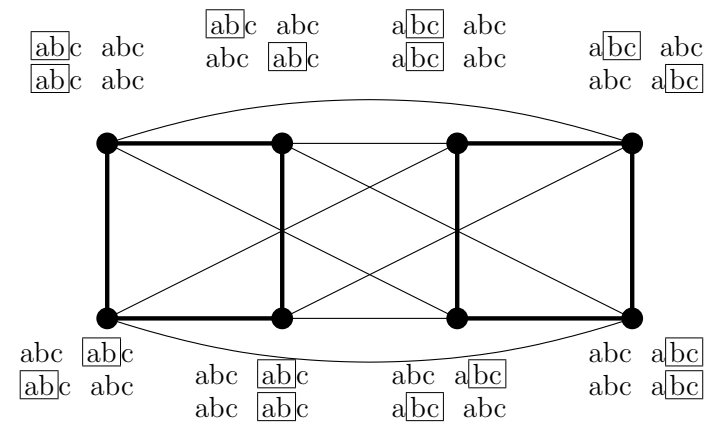

1.2 bc $\overline{a b c}$ ab bc $a b c$ ab $\quad b c$ abc ab $\quad b c$ abc ab

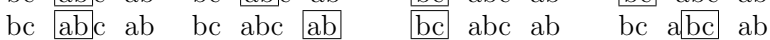

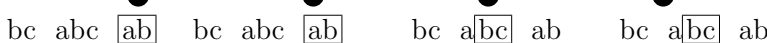
bc $a b c a b$ bc abc ab ac abc ab bc abc ab

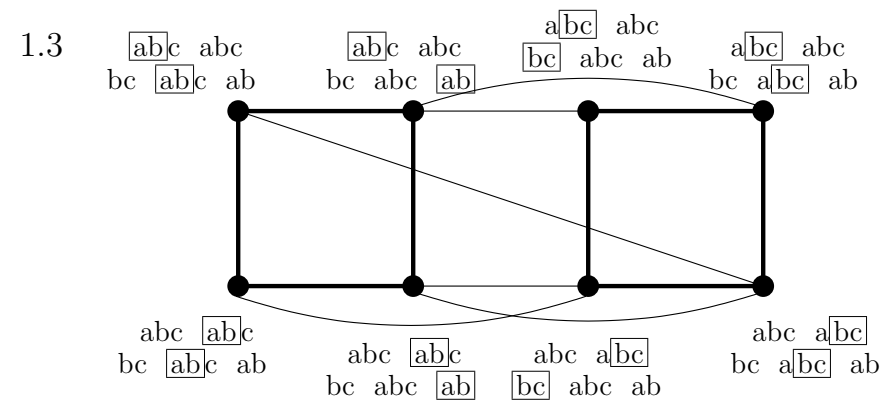

Fig. 3. Subgraphs of $G_{1}$ corresponding to matches of two interfering duos of types (1.1), (1.2) and (1.3). Each node represents a match, e.g., the leftmost upper node in all three subgraphs represents the match between the left occurrence of $a b$ in $\mathcal{A}$ with the left occurrence of $a b$ in $\mathcal{B}$. Bold edges only highlight which conflicts appear between matches of the same duo. Observe that the order of the substrings $a b, b c$ and $a b c$ does not affect the structure of these conflict subgraphs. 
we obey the less breaks we need. A graph $H$ of inconsistent preferences will help us.

By definition, a duo $a b$ without preference has interferences of type (1.1) only, and therefore interferes with at most two other duos. We already observed that if a duo $a b$ has an interference of type (1.1), say with a duo $b c$, and two matches of the duo $b c$ are already fixed, and no new breaks are allowed, then the matches of the duo $a b$ are uniquely given. In this way, a duo without preference transmits a fixed preference of a neighboring duo on one side to a neighboring duo on its other side, etc. A difficulty arises when a preference of one duo, say $b c$, is transmitted by a sequence of duos without preferences to another duo with a preference, say $x y$, and the preference transmitted to $x y$ is different from the preference of $x y$. Then, in every common partition, at least on specific duo $b c$, or $x y$, or one of the transmitting duos, must be a break. We say that the preferences of $b c$ and $x y$ are inconsistent. Similarly, if $b c$ has two different preferences, we also say that $b c$ is inconsistent with $b c$.

We define the graph $H=\left(V_{H}, E_{H}\right)$ of inconsistent preferences. The vertex set $V_{H}$ consists of all duos with a preference and the set of edges $E_{H}$ consists of all pairs of duos with inconsistent preferences (which includes loops for duos inconsistent by themselves). By the above discussion, the graph $H$ can be constructed in time linear in the number of duos.

Lemma 3. The size of a minimum vertex cover for $H$ is a lower bound for the number of breaks in a minimum common partition of $\mathcal{A}_{2}$ and $\mathcal{B}_{2}$.

Proof. Consider a minimum common partition $\pi$ of $\mathcal{A}_{2}$ and $\mathcal{B}_{2}$. By Lemma 1, $\pi$ corresponds to a maximum independent set of $G_{2}$, and thus, also to a minimum vertex cover of $G_{2}$. Let $C_{2} \subseteq V_{2}$ denote nodes in this vertex cover. We observe that for every square $a b$ in $G_{2}$, at least two of its vertices must be in $C_{2}$ and for every square $a b$ with three or four vertices in $C_{2}$, there must be a break between some $a$ and $b$ in $\pi$. Similarly, for every single $c d$ in $C_{2}$, there must be a break between $c$ and $d$ in $\pi$.

We are going to derive a vertex cover $C$ for $H$ from the vertex cover $C_{2}$ for $G_{2}$. The nodes in $C$ will come from three different sources:

- For every square $a b$ with preference in $G_{2}$, if the square $a b$ has three or four vertices in $C_{2}$, we put the vertex $a b \in V_{H}$ to $C$.

- For every single $a b \in C_{2}$, we put $a b \in V_{H}$ to $C$.

- For every square $a b$ without preference in $G_{2}$, if the square $a b$ has three or four vertices in $C_{2}$, we add its closest duo with preference to $C$ (ties broken arbitrarily).

In this way, for every pair of duos with inconsistent preferences, at least one of them will be in $C$, and each vertex in $C$ can be charged to a different break in $\pi$. Thus, $C$ is indeed a vertex cover of size at most equal the number of breaks of the common partition $\pi$.

In phase three, the algorithm cuts all duos corresponding to a minimum vertex cover of $H$ (resp., to 2-approximation of a minimum vertex cover). And 
we charge these cuts to the breaks in the minimum vertex cover, by the above Lemma. Let $\mathcal{A}_{3}$ and $\mathcal{B}_{3}$ denote the two sets of substrings we are left with, and let $G_{3}$ be the corresponding conflict graph.

Lemma 4. The pair $\mathcal{A}_{3}, \mathcal{B}_{3}$ is a common partition of $\mathcal{A}$ and $\mathcal{B}$.

Proof. We are going to construct a maximum independent set in $G_{3}$, corresponding to a common partition of $\mathcal{A}_{3}$ and $\mathcal{B}_{3}$ with no additional breaks. The procedure is based on the the observations in proof of Lemma 3. All we have to do is to note that there are no inconsistent duos in $G_{3}$. Thus, by following the preferences for matches, we will never run into a conflict. If there is a component in $G_{3}$ without any preference, we choose arbitrarily a match for some duo and consider it as a preference.

More specifically, we take to our independent set

- all singles, and,

- from every duo with preference, the two vertices corresponding to the preferred matches, and,

- from every duo without preference the two vertices corresponding to the matches that are forced by a neighboring duo.

In this way, every single from $G_{3}$ appears in the independent set, and for every duo with four possible matches, two of them are in the independent set. This is a maximum independent set corresponding to a common partition with no additional breaks.

The complete algorithm can be schematically summarized as follows.

\section{Algorithm \\ Phase 1: Cut all bad duos. \\ Phase 2: Cut all duos involved in conflicts of type (1.3), (2.1) and (3.1). \\ Phase 3: Cut all duos corresponding to (approximated) minimum vertex cover in the graph $H$ of inconsistent preferences.}

Since the cuts of the algorithm are charged in every phase to different breaks in the optimal solution, at most $4 m$ breaks were used in all three phases, resulting in a 4 -approximation.

Using a hash table, Phases 1 and 2 can be implemented in time $O(n)$. We already noted that the graph $H$ can be constructed in linear time. Since the number of edges in $H$ is $O(n)$, a 2-approximation of the vertex cover can be computed in time $O(n)$, yielding a total time $O(n)$.

For signed MCSP, the algorithm goes along the same lines, it only has to consider $+a+b$ and $-b-a$ as the occurrences of the same duo.

Theorem 5. There exist linear time 4-approximation algorithms for both unsigned and signed $3-\mathrm{MCSP}$. 


\section{Acknowledgment}

We thank Xin Chen for introducing us the MCSP; this motivated our work. We wish to thank Tao Jiang, Marek Chrobak, Neal Young and Stefano Lonardi for many useful discussions. We also gratefully acknowledge comments given to us by Jiří Sgall on early version of the paper.

\section{References}

1. A. Avidor and U. Zwick. Approximating MIN $k$-SAT. In Proceedings of 13th International Symposium on Algorithms and Computation (ISAAC), volume 2518 of Lecture Notes in Computer Science, pages 465-475, 2002.

2. P. Berman and M. Karpinski. On some tighter inapproximability results. In Proceedings of the of 26th International Colloquium on Automata, Languages and Programming, volume 1644 of Lecture Notes in Computer Science, pages 200-209, 1999.

3. A. Caprara. Sorting by reversals is difficult. In Proceedings of the First International Conference on Computational Molecular Biology, pages 75-83, 1997.

4. X. Chen, J. Zheng, Z. Fu, P. Nan, Y. Zhong, S. Lonardi, and T. Jiang. Assignment of orthologous genes via genome rearrangement. Submitted, 2004.

5. D. A. Christie and R. W. Irving. Sorting strings by reversals and by transpositions. SIAM Journal on Discrete Mathematics, 14(2):193-206, 2001.

6. M. Chrobak, P. Kolman, and J. Sgall. The greedy algorithm for the minimum common string partition problem. In Proceedings of the 7th International Workshop on Approximation Algorithms for Combinatorial Optimization Problems (APPROX), 2004.

7. G. Cormode and S. Muthukrishnan. The string edit distance matching problem with moves. In Proceedings of the 13th Annual ACM-SIAM Symposium On Discrete Mathematics (SODA), pages 667-676, 2002.

8. M. R. Garey and D. S. Johnson. Computers and Intractability: A Guide to the Theory of NP-Completeness. W.H. Freeman \& Company, San Francisco, 1978.

9. S. Hannenhalli and P. A. Pevzner. Transforming cabbage into turnip: polynomial algorithm for sorting signed permutations by reversals. Journal of the ACM, 46(1):1-27, Jan. 1999.

10. D. Sankoff and N. El-Mabrouk. Genome rearrangement. In T. Jiang, Y. Xu, and M. Q. Zhang, editors, Current Topics in Computational Molecular Biology. The MIT Press, 2002.

11. D. Shapira and J. A. Storer. Edit distance with move operations. In 13th Symposium on Combinatorial Pattern Matching (CPM), 2002.

12. G. A. Watterson, W. J. Ewens, T. E. Hall, and A. Morgan. The chromosome inversion problem. Journal of Theoretical Biology, 99:1-7, 1982. 